Apidologie, 1979, 10 (2), 115-128.

\title{
BEITRAG ZUR KENNTNIS DER ALTERSVERÄNDERUNGEN IM NERVENSYSTEM UND IN ANDEREN INNEREN ORGANEN DER BIENENKÖNIGIN (APIS MELLIFERA L.)
}

\author{
Étude des modifications apportées par l'âge \\ au système nerveux et à d'autres organes internes \\ de la reine d'abeille (Apis mellifera $\mathbf{L}$.)
}

\author{
W. FYG* \\ Bubenbergstrasse 27 - CH - 3700 Spiez \\ SUMMARY \\ STUDY OF THE CHANGES DUE TO AGE IN THE NERVOUS SYSTEM \\ AND IN OTHER INTERN ORGANS \\ OF THE HONEYBEE QUEEN ( $A P I S$ MELLIFERA)
}

The incidence of amyloid in the internal organs of ageing honeybee queens is described. These glyco-protein compounds are found as microscopically small and frequently agglomerating bodies in various tissues, in intra- and extracellular position; they are easily demonstrated by specific staining methods.

Amyloid deposits were never found in young queens so far, but in all elder, mostly healthy queens. The quantity of amyloid in the affected organs - nervous system, wall of the heart, spermatheca and certain glands - is evidently increasing with age. Thus the amyloic degeneration of the tissues seems to be a normal, characteristic process of ageing. Moreover different other alterations correlated with increasing age, found in internal organs of queen bees, are described.

A causal connection is supposed between the amyloidosis of the neurosecretory cells in the cerebral ganglion and in the corpora cardiaca, and the diminished production of queen substance in old queens; further, between the amyloid bodies in the epithelial cells of the spermatheca and the laying of drone brood only by an old queen.

\section{ZUSAMMENFASSUNG}

Es wird das Vorkommen von Amyloid in den inneren Organen der alternden Bienenköniginnen beschrieben. Diese kohlenhydrathaltige Eiweissverbindung findet sich in Form von mikroskopisch kleinen,

* Dr. h.c. Werner FxG ist während der Drucklegung dieses Beitrages am 1.4.79 verstorben. Er wird als hervorragender Spezialist für die Krankheiten und Anomalien der Bienenkönigin in der Fachwelt unvergessen bleiben. 
oft agglomerierenden Körperchen intra- und extrazellulär in den verschiedensten Geweben und lässt sich mit indikatorischen Färbemethoden in Nativ- und Schnittpräparaten leicht nachweisen. Die amyloiden Ablagerungen wurden bis jetzt nie in jungen, dagegen bei allen älteren, grösstenteils gesunden Königinnen festgestellt. Da der Amyloidgehalt der betroffenen Organe, insbesondere des Nervensystems, der Herzwand, der Samenblase und gewisser Drüsen mit fortschreitendem Alter offensichtlich zunimmt, dürfte es sich bei dieser amyloiden Entartung der Gewebe um einen normalen, sehr charakteristischen Alterungsprozess handeln. Ausserdem wird auf verschiedene andere Altersveränderungen hingewiesen, die an den inneren Organen der Bienenköniginnen zu beobachten sind.

\section{EINLEITUNG}

Die Honigbiene ist schon wiederholt zum Studium der Altersveränderungen an den inneren Organen benützt worden, weil sich dieses soziale, unter natürlichen Bedingungen lebende Insekt für derartige Untersuchungen sehr gut eignet. Ein Fachmann kann sich bei Imkern oder in Bieneninstituten alle benötigten Altersstadien der Biene in fast beliebiger Zahl leicht beschaffen. Es stehen fast jederzeit Tiere der verschiedensten Altersstufen zur Verfügung: Höchstalter der Arbeiterinnen im Sommer etwa sechs Wochen, im Winter sechs bis acht Monate; Lebensdauer der Königinnen drei bis fünf Jahre.

Von den älteren Abhandlungen verdient vor allem diejenige von WEYER (1931) ein besonderes Interesse. Er hat festgestellt, dass im Bienengehirn mit zunehmendem Alter auffallende Veränderungen stattfinden. Während bei jungen Bienen die Zellstrukturen sehr regelmässig sind, lockert sich ihr Gefüge im Alter mehr und mehr auf. Im Cytoplasma der Ganglienzellen treten viele, stark färbbare Granula auf und das Chromatin ihrer Kerne ballt sich zusammen. Auch die Medullar- oder Fibrillärsubstanz zeigt Strukturänderungen, indem die Fasermassen mehr oder minder schrumpfen, wobei sich zwischen den Nervenfaserbündeln kleinere und grössere Spalten bilden. Die ersten Anzeichen einer zerebralen Degeneration treten nach den Beobachtungen von WEYER gewöhnlich bei fünf bis sechs Wochen alten Arbeitsbienen auf und zwar ganz unabhängig von ihrer Tätigkeit und der Jahreszeit. Von diesem Zeitpunkt an verlaufen dann die Degenerationsprozesse in den verschiedenen Hirnbezirken meistens sehr rasch. Entgegen der Ansicht von Schмidt (1923) widerstehen die Ganglienzellen in den als assoziative Zentren funktionierenden Corpora pedunculata der Degeneration am längsten. Besondere Beachtung verdient die Feststellung von WEYER, dass die soeben erwähnten, altersbedingten Strukturänderungen im Gehirn von Biene zu Biene stark variieren und weitgehend von der jeweils angewandten Fixierung des Materials abhängig sind. Seiner Meinung nach ist die zerebrale Degeneration aber nicht die primäre Ursache der früher oder später eintretenden körperlichen Dekadenz und des natürlichen Todes der Biene; beides ist eher auf eine allgemeine und viele Organe in Mitleidenschaft ziehende Stoffwechselstörung zurückzuführen. 
Eine andere zerebrale Degenerationserscheinung hat LuChtBertram (1963), beschrieben, nämlich die Bildung von kugeligen und schlauchförmigen Hohlräumen (Fig. 1) im Gehirn von alten Bienenköniginnen. Sie finden sich in verschiedenen Hirnbezirken, vorwiegend aber im Bereich der Ganglienzellen der Seh- und Riechlappen, sowie im Unterschlundganglion. Diese Hohlräume sollen beim altersbedingten Absterben und der Autolyse einer grösseren Zahl von Ganglienzellen entstehen. Die eigenen Untersuchungen ergaben demgegenüber, dass solche Gewebelücken im Zentralnervensystem nicht nur bei mehrjährigen, sondern gar nicht so selten auch bei jungen Bienenköniginnen vorkommen (Tab. 1). Obwohl ihre Entstehungsweise noch ungenügend bekannt ist, steht jedenfalls fest, dass wir es dabei nicht mit einem eindeutigen Alterskennzeichen zu tun haben. Von den 62 untersuchten Königinnen wiesen $48(=77,4 \%)$ keinerlei Krankheitszeichen auf.

Im Verlaufe der Untersuchung zahlreicher Königinnen verschiedenen Alters an der Bienenabteilung der Eidgenössischen Milchwirtschaftlichen Forschungsanstalt Liebefeld hat sich dagegen die mit dem Alter zunehmende Einlagerung von Amyloid (*) in verschiedenen inneren Organen der Bienenkönigin als charakteristische Altersveränderung erwiesen. Amyloid-Einlagerungen im alternden Gewebe des Menschen und höherer Wirbeltiere sind schon seit VIRCHOW bekannt (SCHNEIDER 1964, SchwarTz 1966). Da der Amyloid-Nachweis sehr rasch zu führen ist, bietet er sich als eine einfache Methode zum Aufzeigen von Altersveränderungen an, zumal diese Einlagerungen meist in mehreren Organen gleichzeitig auftreten (zusätzlich zu organspezifischen Altersveränderungen) (FYG 1960, 1961, 1965, 1966, 1967).

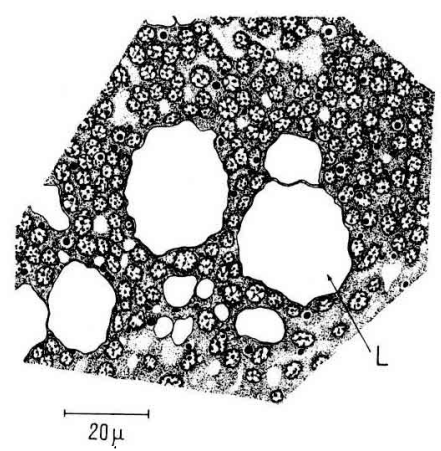

ABB. 1. - Lochbildungen im Lobus opticus einer dreijährigen Bienenkönigin. L Hohlraum.

Fig. 1. - Formation de lacunes dans le lobe optique d'une reine âgée de trois ans. $\mathrm{L}$ Lacune.

(*) Amyloid gehört zu den Glykoproteiden. 
TAB. 1. - Hohlraumbildungen im ZNS von Bienenköniginnen verschiedenen Alters.

TABL. 1. - Formations de lacunes dans les cellules neurosécrétrices de reines d'abeille d'âge divers.

\begin{tabular}{|c|c|c|}
\hline \multirow{3}{*}{$\begin{array}{c}\text { Alter der Bienenköniginnen } \\
\text { Age des reines }\end{array}$} & \multicolumn{2}{|c|}{$\begin{array}{l}\text { Ober- und Unterschlundganglion } \\
\text { Ganglion sus- et sous-œsophagien }\end{array}$} \\
\hline & $\begin{array}{c}\text { ohne } \\
\text { sans }\end{array}$ & $\begin{array}{l}\text { mit } \\
\text { avec }\end{array}$ \\
\hline & \multicolumn{2}{|c|}{$\begin{array}{c}\text { Lochbildungen } \\
\text { formation de lacunes }\end{array}$} \\
\hline $\begin{array}{l}\text { weniger als } 1 \text { Jahr alt } \ldots \ldots \ldots \ldots \ldots \ldots \ldots \\
\text { moins d'un an }\end{array}$ & 12 & 7 \\
\hline $\begin{array}{l}\text { einjährig } \ldots \ldots \ldots \ldots \ldots \ldots \ldots \ldots \ldots \ldots \ldots \\
\text { un an }\end{array}$ & 0 & 11 \\
\hline $\begin{array}{l}\text { zweijährig } \ldots \ldots \ldots \ldots \ldots \ldots \ldots \ldots \ldots \\
\text { deux ans }\end{array}$ & 0 & 9 \\
\hline $\begin{array}{l}\text { dreijährig } \ldots \ldots \ldots \ldots \ldots \ldots \ldots \ldots \ldots \ldots \\
\text { trois ans }\end{array}$ & 0 & 10 \\
\hline $\begin{array}{l}\text { vierjährig } \\
\text { quatre ans }\end{array}$ & 0 & 6 \\
\hline $\begin{array}{l}\text { fünfjährig } \ldots \ldots \ldots \ldots \ldots \ldots \ldots \ldots \ldots \ldots \\
\text { cinq ans }\end{array}$ & 0 & 2 \\
\hline $\begin{array}{l}\text { alte Königinnen ohne genaue Altersangaben } \ldots \ldots \\
\text { reines âgées, sans précision d'âge }\end{array}$ & 0 & 5 \\
\hline
\end{tabular}

\section{MATERIAL UND METHODE}

Es wurden histologische Präparate von 97 Bienenköniginnen hergestellt, die zur diagnostischen Untersuchung mit verschiedener Fragestellung eingesandt worden waren.

Spezifische Färbemethoden zum Amyloidnachweis :

\section{Jodreaktion}

Behandlung mit Lugolscher Jodjodkaliumlösung bis zu einer schwachen Gelbfärbung.

Nachbehandlung mit verdünnter Schwefelsäure Amyloidkörper tief dunkelblau bis dunkelviolett (Abb. 2). Gelingt sowohl an Schnitten wie an frischem Gewebe.

\section{PAS-Reaktion (Perjodsäure-Schiff-Reagens)}

\section{Karminfärbung nach Best}

Fixierung mit Carnoy- oder alkoholischem Pikrinsäuregemisch.

Vorbehandlung mit $1 \%$ Diastase, mindestens zwei Stunden (zur Entfernung des Glykogens).

Färbung mit Best'schem Karmin (s. Romeis 1948).

Nachfärbung mit Weigertschem Eisenhämatoxylin.

Ergebnis : Sehr kontrastreiche, spezifische Darstellung der Amyloidkörper (Abb. 3).

Fluoreszenzmikroskopische Verfahren wurden für diese Untersuchung nicht angewandt. 


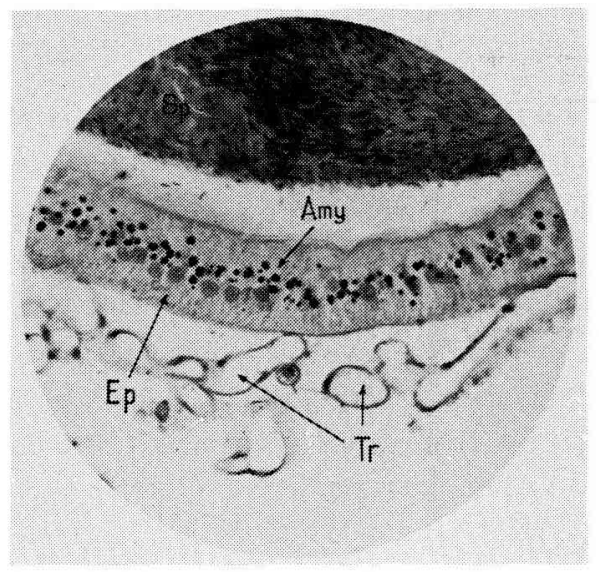

ABB. 2. - Amyloid in der Samenblasenwand einer dreijährigen Bienenkönigin. Jodjodkalium - Schwefelsäure - Reaktion.

Amy Amyloid; Ep Epithelzellen; Tr Trachealhülle.

FIG. 2. - Amyloïde dans la paroi de la spermathèque d'une reine âgée de trois ans. Réaction iode-iodure de potassium - acide sulfurique (amyloïde en noir). Amy amyloïde; Ep épithélium; Tr trachées.

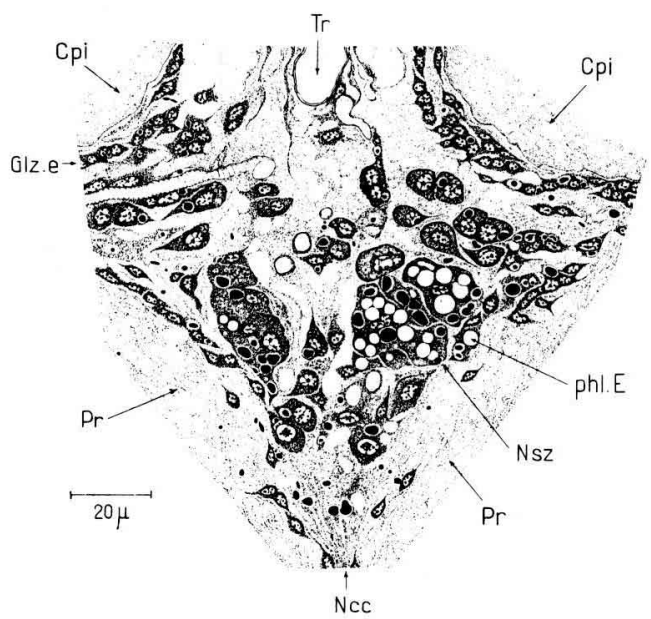

ABв. 3. - Amyloid in den neurosekretorischen Zellen der Pars intercerebralis einer dreijährigen Bienenkönigin (Frontalschnitt; Amyloid schwarz, phloxinophile Einschlüsse weiss).

Cpi Pilzkörper (Corpora pedunculata), innerer Becher;

Glz.e. Pilzkörper, Globulizellen; Ncc Nervi corporis cardiaci;

NsZ Neurosecretorische Zellen; Phl. E. phloxinophile Einschlüsse; Pr Protocerebrum; Tr Tracheen.

FiG. 3. - Amylö̈de dans les cellules neurosécrétrices du cerveau d'une reine âgée de trois ans.

(Coupe frontale; amyloïde en noir, inclusions phloxinophiles en blanc).

Cpi Calix interne; Glz.e. cellules globulaires externes des corps pédonculés; Ncc nerfs du corpus cardiaca;

NsZ cellules neurosécrétrices; Phl.E. inclusions phloxinophiles; Pr protocérébron; Tr trachées. 


\section{ALTERSABHÄNGIGKEIT UND LOKALISATION DER AMYLOIDABLAGERUNGEN}

Bei 13 jungen, weniger als ein Jahr alten Königinnen wurden weder am Nervensystem noch in Drüsen oder anderen inneren Organen Amyloidkörper gefunden.

Nach der ersten Überwinterung, also im Alter von etwa einem Jahr, traten bei etwa der Hälfte der Königinnen in verschiedenen Geweben kleine Amyloidtropfen auf. Die individuelle Schwankung ist un diese Zeit sehr beträchtlich.

Die Grösse der Amyloidkörper wechselt mit dem Lebensalter. Bei zweijährigen und älteren Königinnen sind die Körper regelmässig zu finden (untersucht wurden sechs zweijährige und 18 drei- bis fünfjährige Königinnen). Bei den mehr als dreijährigen Königinnen können die Amyloidkörper einen Durchmesser von $10 \mu \mathrm{m}$ und mehr erreichen (Abb.4). In vielen amyloiden Gebilden wird nach dem Behandeln mit Lugolscher Lösung und Säure eine konzentrische Schichtung sichtbar, wie sie auch von bestimmten Corpora amylacea in manchen menschlichen Organen bekannt ist (BORST 1950).

Wie auf den Abb. 2-4 zu erkennen ist, liegen die Amyloidkörper sowohl intrazellulär wie in den interzellulären Räumen.

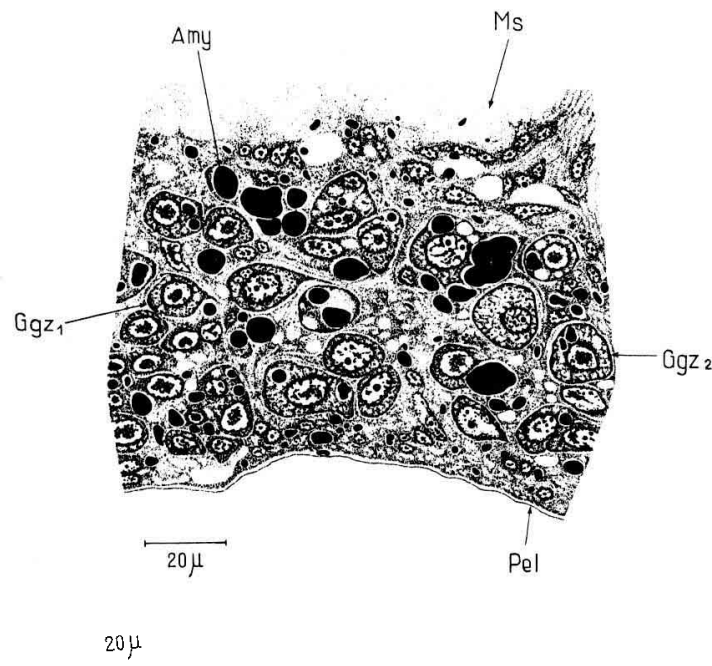

Aвв. 4. - Amyloidablagerungen in der ventralen Ganglienzellenschicht des Unterschlundganglions einer vierjährigen Bienenkönigin.

Frontalschnitt (Amyloid schwarz).

Amy Amyloid; $\mathrm{Ggz}_{1}$ Ganglienzellen mit verklumptem Kernchromatin; $\mathrm{Ggz}_{2}$ Ganglienzellen mit intrazellulärem Amyloid; Ms Mark- oder Medullarsubstanz; Pel Perilemm.

FIG. 4. - Dépôts d'amyloïde dans la couche ventrale des cellules ganglionnaires du ganglion sous- asophagien d'une reine âgée de quatre ans. Coupe frontale (amyloïde en noir)

Amy amyloïde; $\mathrm{Ggz}_{1}$ cellules ganglionnaires avec la chromatine en motte;

$\mathrm{Ggz}_{2}$ cellules ganglionnaires avec amyloïde intracellulaire;

Ms substance fibreuse ou masse fibrillaire; Pel névrilemme. 
Im Zeitpunkt des Auftretens und den Prädilektionsstellen gibt es - bei aller individuellen Variabilität - eine auffällige Regelmässigkeit, die allein aufgrund des Ausmasses und der Lokalisation der Ablagerungen eine Altersdiagnose der Königin erlaubt.

Im Nervensystem waren die ersten Amyloidkörper bei drei von fünf einjährigen Königinnen als kleine, kugelige Gebilde von unterschiedlicher Grösse in und zwischen den Ganglienzellen der Pars intercerebralis des Protocerebrums, in den hier liegenden neurosekretorischen Zellen (Abb. 3) und in der Rindenschicht des Deutocerebrums sowie des Subösophagealganglions (Abb. 4 u. 5) zu finden. Die intrazellulären Amyloidkörperchen liegen im Cytoplasma vorwiegend in der Nähe der Zellperipherie.

In den Lobi optici, den Pilzkörpern und in der Medullarsubstanz fehlen die Einlagerungen in dieser Phase noch gänzlich. Erst bei dreijährigen Königinnen finden sich Amyloidkörper in allen Abschnitten des Nervensystems, einschliesslich des Bauchmarks. In den plasmaarmen assoziativen Ganglienzellen der Corpora pedunculata treten solche Amyloidablagerungen als sehr kleine Kügelchen ebenfalls, aber erst im höheren Alter auf, und zwar vornehmlich in den äusseren, den Becherwandungen anliegenden Zellgruppen, während die zentralen Neuren davon frei bleiben. Dieser Befund stimmt mit den Beobachtungen von WEYER (1931) überein, wonach auch bei der Arbeitsbiene gerade diese Assoziationszentren der altersbedingten Entartung am längsten widerstehen und mit seltenen Ausnahmen noch intakt bleiben, wenn die grossen Ganglienzellen der Pars intercerebralis schon stark degenerative Veränderungen zeigen.

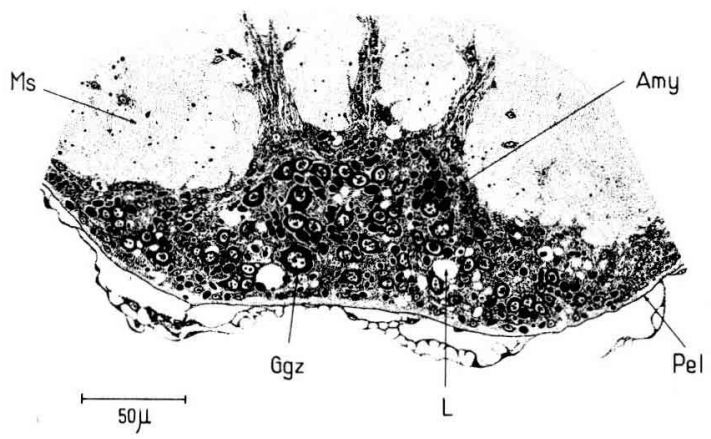

ABB. 5. - Frontalschnitt durch die ventrale Rindenschicht des Unterschlundganglions einer fünfjährigen Bienenkönigin. (Amyloidablagerungen schwarz) Amy Amyloid; L Lochbildung im Lobus opticus : Ms Mark- oder Medullarsubstanz; Pel Perilemm; Ggz Ganglienzellen.

FIG. 5. - Coupe frontale à travers la couche corticale de la zone ventrale du ganglion sous-asophagien d'une reine âgée de cinq ans.

(Amyloïde en noir) Amy amyloïde; L lacune du lobe optique; Ms substance fibreuse ou masse fibrillaire;

Pel névrilemme; Ggz cellules ganglionnaires ou nerveuses. 
Bemerkenswert ist die Feststellung, dass bei mindestens dreijährigen Königinnen die Ablagerungen nicht nur in den Ganglien, einschliesslich des stomatogastrischen Nervensystems, sondern auch in den peripheren Nerven (Abb. 6) auftreten. Sie liegen hier teils dicht unter der feinen Hüllschicht, teils zwischen den feinen Fasern. Diese Befunde sprechen jedenfalls dafür, dass sich die altersbedingten amyloiden Degenerationsprozesse, die mit den drei vorerwähnten Methoden nachgewiesen werden können, nicht nur im zentralen, sondern offenbar im ganzen Nervensystem abspielen.

Im endokrinen System sind die ersten Einlagerungen, wie schon oben erwähnt, in und zwischen den neurosekretorischen Zellen der Pars intercerebralis zu finden (Abb. 3). Mit steigendem Alter nehmen die Amyloidkörper rasch an Zahl und Grösse zu. Bei der Karminfärbung mit vorangehender Diastasebehandlung sind sie eindeutig von den ebenfalls mit dem Alter zunehmenden acidophilen Zelleinschlüssen zu differenzieren, die von PfLugfelder (1958) und BiedermanN (1964) ebenfalls als Altersveränderung aufgefasst wurden. Nach meinen eigenen Präparaten handelt es sich bei dieser Substanz, die sich mit Phloxin leuchtend rot färbt, jedoch um das Sekret der Drüsenzellen.

Im retrocerebralen Hormondrüsensystem, das bei der Königin wesentlich stärker entwickelt ist als bei den Arbeiterinnen, zeigen nur die Corpora cardiaca und die von ihnen abhängenden Nervi corporis allati bei alten Königinnen Amyloideinschlüsse, nicht aber die Corpora allata, von den Hili abgesehen (Abb. 7).

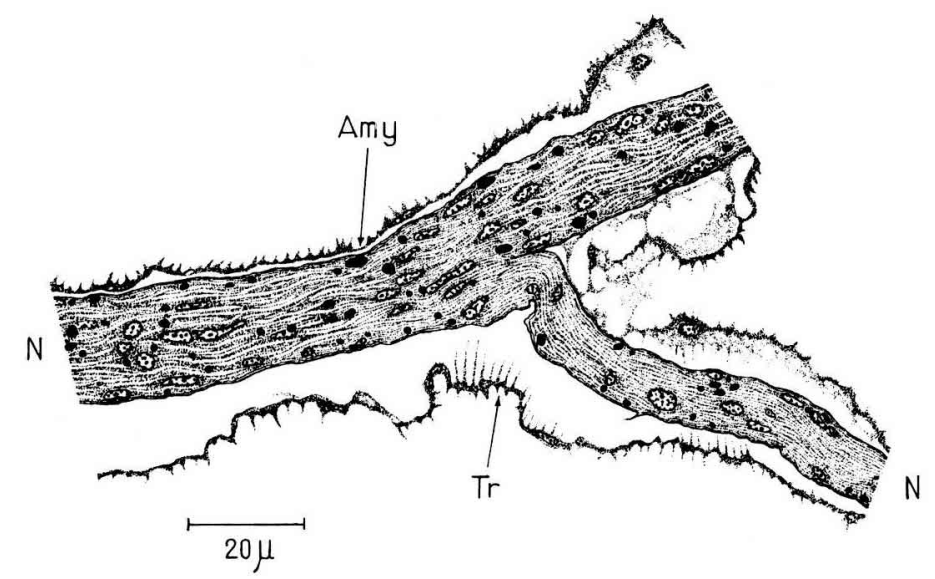

Aвв 6. - Amyloidkörperchen (schwarz) im Labralnerv einer dreijährigen Bienenkönigin. N Nerv; Tr Tracheen.

FIG. 6. - Corpuscules amyloïdes (en noir) dans le nerf labral d'une reine âgée de trois ans. $\mathrm{N}$ nerf; $\mathrm{Tr}$ trachées. 


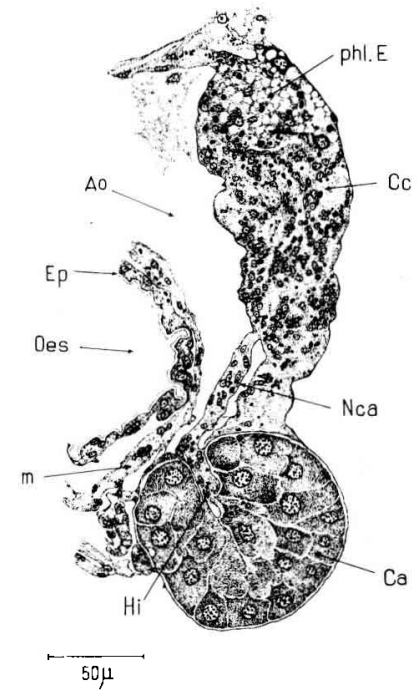

Aвв. 7. - Corpus cardiacum und Corpus allatum einer dreijährigen Bienenkönigin.

Frontalschnitt (Amyloidablagerung schwarz)

(Ao Aorta; C.a. Corpus allatum; C.c. Corpus cardiacum;

Ep Epithel; Hi Hilus; m Muskelfasern; Nca Corpus-allatum-Nerv;

Oes Oesophagus; phl.E phloxinophile Einschlüsse.

FIG. 7. - Corpus cardiacum et corpus allatum d'une reine agée de trois ans.

Coupe frontale (amyloïde en noir)

Ao aorte; C.a. corpus allatum; C.c. corpus cardiacum;

Ep épithélium; Hi hilus; $m$ fibres musculaires; Nca nerf allate; Oes œsophage; phl.E. : inclusions phloxinophiles.

In den Mandibeldrüsen wurden in den Drüsenzellen selbst keine Amyloidkörper gefunden, wohl aber - manchmal in erheblichem Ausmass - in den begleitenden Fettzellen (Abb. 8). Da zwischen den Fett- und Drüsenzellen sehr wahrscheinlich enge funktionelle Beziehungen bestehen (ÖRÖsı-PAL 1968), ist nicht auszuschliessen, dass die bei alten Königinnen feststellbare Verkleinerung der Drüsenzellen und ihrer Kerne eine Folge der amyloiden Entartung der Fettzellen ist.

Im Darmtrakt zeigen die Rektalpapillen besonders auffällige Altersveränderungen, die sich vor allem in einer bräunlichen Verfärbung und einer Degeneration des Innenepithels mit Vakuolenbildung manifestieren (FYG 1956). Gleichzeitig kommt es im Aussenepithel zur Einlagerung zahlreicher Amyloidkörper. Dasselbe ist im Pylorusepithel festzustellen (FYG 1967). Dagegen wurde bezeichnenderweise in dem sich ständig regenerierenden Mitteldarmepithel nie Amyloid gefunden (in den Muskelfasern des Mitteldarms dagegen treten zahlreiche Einschlüsse auf).

Von den Anhängen des Darmtraktes zeigen die Hypopharyngeal- und die Labialdrüsen mit dem Alter zunehmende Amyloideinschlüsse, beginnend schon bei manchen einjährigen Königinnen. 


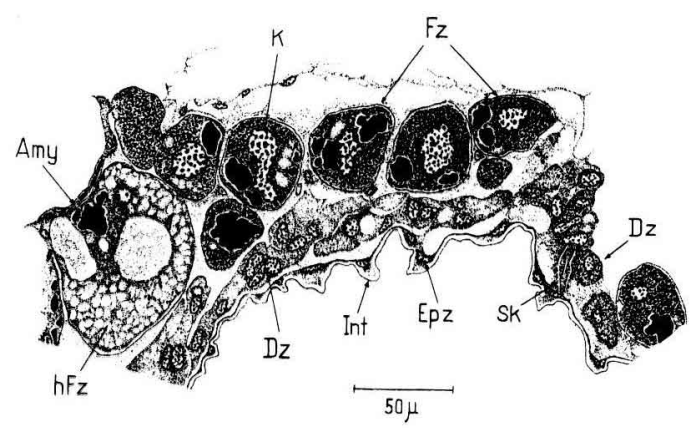

Авв. 8. - Ausschnitt aus der Mandibeldrüsenwand einer alten, greisenbrütigen Bienenkönigin.

Amy Amyloid, Dz Drüsenzelle; Epz Epithelzellen; $\mathrm{Fz}$ Fettzellen; hFz hypertrophierte Fettzelle; Int Intima des Drüsenhohlraumes; K Zellkern; Sk Sekretkanälchen.

FIG. 8. - Coupe à travers la paroi de la glande mandibulaire d'une reine âgée et devenue bourdonneuse. Amy amyloïde; Dz cellules glandulaires;

Epz cellules épithéliales; $F_{z}$ cellules adipeuses; $h F_{z}$ cellule adipeuse hypertrophiée Int cuticule interne; $\mathrm{K}$ noyau de cellule; Sk canalicules excréteurs.

Die Malpighischen Gefässe gehören mit den Rektalpapillen zu den Organen, deren Altern schon im Nativzustand an einer Farbveränderung zu erkennen ist (FYG 1963). Bei den frisch geschlüpften und ganz jungen Bienenköniginnen sind sie farblos und transparent. Im Verlaufe des ersten Lebensjahres werden sie in der Regel gelblich, im zweiten gelb bis gelbgrün und vom dritten Jahr hinweg grün bis intensiv dunkelgrün. Diese charakteristische Verfärbung der Malpighi'schen Gefässe, die eine recht zuverlässige Altersschätzung ermöglicht, wird durch die Ablagerung von verschiedenartigen, teils pigmentierten Stoffwechselschlacken verursacht. Jedenfalls findet man bei allen alternden Bienenköniginnen in den Epithelzellen neben dem normalerweise vorkommenden Glykogen fein- bis grobkörnige Konkremente von unterschiedlicher Farbe, die hier offenbar deponiert und nicht ausgeschieden werden. Abgesehen von kleinen Glykogenpartikeln enthalten die Malpighi'schen Gefässe der gesunden Königinnen im Lumen nie geformte Exkrete; nur in pathologischen Fällen kommt das vor (Fyg 1963). Über die chemische Natur der im Harngefässepithel abgelagerten Stoffe und Pigmente ist noch sehr wenig bekannt. Eine Ausnahme davon macht eigentlich nur das Amyloid, welches bei vielen älteren Bienenköniginnen in wechselnder Menge in den Epithelzellen nachgewiesen werden kann. Besonders reichlich findet es sich oft in den mittleren und unteren Abschnitten der Gefässe in Form von kleineren und grösseren Agglomeraten. Die Malpighi'schen Gefässe gehören somit ebenfalls zu den Organen, die mit zunehmendem Alter amyloid entarten.

Im Kreislaufsystem wurden bei älteren Königinnen in den Muskelzellen Amyloidkörper gefunden, nicht aber im Diaphragma oder in Haemocyten. 
Ähnlich auffällig wie in den Malpighi'schen Gefässen sind die Altersveränderungen im Speichergewebe des Fettkörpers. In den Trophocyten ist fast regelmässig eine mit dem Alter zunehmende Amyloidose festzustellen. In den Oenocyten hingegen treten keine Amyloidkörper, aber schon bald nach dem Schlüpfen feinkörnige Einschlüsse auf, die ihre Farbe mit der Zeit von blassgelb zu dunkelgelb bis bräunlich verändern. Im höheren Alter erscheint deshalb der Fettkörper mehr oder weniger braun gesprenkelt. Uber die chemische Natur dieser Einschlüsse in den Oenocyten ist $\mathrm{m}$.W. bisher noch nichts bekannt.

Eigenartigerweise wurde im Epithel der Giftdrüse und der Giftblase, das schon frühzeitig deutliche Degenerationszeichen aufweist (Schrumpfung, Verfärbung und Verhärtung des Gewebes und des Sekretes), keine Amyloidkörper gefunden. Diese beschränken sich im Stachelapparat auf die Muskulatur und die Dufour'sche Drüse.

An den Fortpflanzungsorganen der Bienenkönigin manifestieren sich die Altersveränderungen in zwei offenbar voneinander unabhängigen Prozessen :

1. Eine früh einsetzende und fortschreitende Degenerationsverkalkung in der Valvula vaginalis, die an anderer Stelle eingehend beschrieben wurde (Fyg 1966).

2. Die mit dem Alter zunehmende Ablagerung von Amyloid im Epithel der Spermatheka (Abb. 2) und - erst im höheren Alter - in der Muskulatur des Ductus spermaticus.

Obwohl ich viele junge, nur wenige Monate alte Bienenköniginnen untersucht habe, konnten in ihrem Samenblasenepithel nie Amyloidkörperchen festgestellt werden. Aber bereits bei einjährigen Tieren zeigen sich in diesem Gewebe die ersten solcher Gebilde. Sie sind freilich noch sehr klein und liegen meist in der Nähe der Zellkerne in deutlichen Vakuolen. Mit dem Älterwerden der Königinnen nimmt ihre Zahl und Grösse rasch zu (Abb. 2, Amy), so dass sie selbst in Nativpräparaten dank ihrer Lichtbrechung als hyaline Körperchen ohne weiteres zu erkennen sind. Sie können schliesslich so reichlich vorhanden sein, dass die Samenblasenwand davon völlig durchsetzt erscheint.

In geringerem Masse wurde Amyloid auch in der Wand von Ovidukten und Vagina gefunden, nicht aber in den Ovarien und in den Spermathekaldrüsen.

\section{DISKUSSION}

In Körpergeweben von Bienenköniginnen sind eine Reihe von Altersveränderungen festzustellen, so degenerative Zellveränderungen, Schrumpfungsvorgänge, Einlagerung von Glykogen und pigmentierten Körnern unbekannter Zusammensetzung. Zu diesen Einlagerungen gehören auch intra- und extrazellulär gelegene Amyloidkörner, die mit histochemischen Methoden sehr einfach nachzuweisen sind.

Bei jungen Königinnen (vor der ersten Überwinterung) wurden Amyloidkörper niemals gefunden. 
Bei alten Königinnen treten sie hingegen ziemlich regelmässig auf, und zwar mit zunehmendem Alter in verstärktem Masse (sowohl nach Anzahl wie nach Grösse). Sehr viele dieser Königinnen waren noch voll leigstungsfähig und liessen keine Krankheitszeichen erkennen.

Aus einer Ưbersicht über ein grosses Untersuchungsmaterial lässt sich keine Beschränkung auf einen bestimmten Gewebetyp erkennen. Wohl aber sind einzelne Organe oder Organsysteme fast regelmässig befallen, andere wieder nach den vorliegenden Untersuchungen niemals.

$\mathrm{Zu}$ den regelmässig befallenen Organen gehört das Nervensystem (zentral und peripher), das endokrine System (aber mit Ausnahme der Corpora allata), das Drüsensystem des Darmtrakts (einschliesslich der Rektalpapillen und der Malpighischen Gefässe), ein Teil der Muskulatur (Herz, Ovidukte, Samenblasengang, Stachelapparat), die Fettzellen und das Epithel der Spermatheka.

Ohne Einschlüsse bleiben auch bei alten Königinnen Darmepithel, Giftdrüsen, Spermathekaldrüsen und Corpora allata.

Lokale Amyloidablagerungen oder generalisierte Amyloidosen sind seit langem auch bei Vertebraten und insbesondere beim Menschen als Altersveränderungen bekannt. Man nimmt an, dass die Amyloidose auf einer Störung des Eiweissstoffwechsels beruht, wobei offenbar ein gelöstes pathologisches und kohlenhydrathaltiges Eiweiss im Blute kreist und nach seinem Durchtritt durch die Gefässwände in den Geweben, insbesondere in den Grundsubstanzen des Bindegewebes als Gel ausfällt (SCHNEIDER 1964).

Es muss dahingestellt bleiben, ob die Amyloideinlagerungen bei der Bienenkönigin lediglich eine von mehreren Altersveränderungen ohne spezifische Bedeutung darstellen, oder ob sie zu bestimmten Ausfällen führen. Alte Königinnen verschwinden gewöhnlich eines Tages aus dem Volk, ohne dass man sagen könnte, ob sie aus einer bestimmten Ursache gestorben wären oder ob sie von den Arbeiterinnen beseitigt wurden. Es gibt jedoch zwei Fälle, in denen der Ausfall der Königin mit Störungen in einem bestimmten Organ, bzw. Organsystem in Verbindung gebracht werden kann :

1. Alte Königinnen werden häufig von den Arbeiterinnen als minderwertig " erkannt ». Es kommt dann zur Aufzucht junger Königinnen, die dann früher oder später die alte Königin beseitigen (stille Umweiselung). Nach ButLER (1960) beruht dieses Verhalten der Arbeiterinnen auf einer ungenügenden Produktion von " queen substance " durch die gealterte Königin.

2. Alte Königinnen legen nicht selten teilweise oder ausschliesslich unbefruchtete Eier, aus denen sich Drohnen entwickeln ("Greisenbrütigkeit »). Als Ursache wurde eine Inaktivierung der Spermatozoen in einer nicht mehr funktionstüchtigen Spermatheka festgestellt (FYG 1960).

In beiden Fällen kann ein enger Zusammenhang mit der Amyloidablagerung festgestellt werden. Wie oben angeführt, sind bei älteren Königinnen besonders viele 
und grosse Amyloidkörper in und zwischen den Zellen des neurosekretorischen Systems und der Corpora cardiaca gefunden worden. Der Zusammenhang dieses Hormonsystems mit der Fortpflanzung ist seit langem bekannt (BiedermanN 1964, HERRMANN 1969). Bei greisenbrütigen Königinnen wurde Amyloid in beträchtlichen Mengen in den Epithelzellen der Spermatheka gefunden (FYG 1960), deren Ausschaltung zu einer Inaktivierung der Spermatozoen in der Samenblase führt (G. KoENiger 1970).

\title{
DANK
}

Herrn Prof. Dr. F. RUtTNeR, Oberursel, danke ich für die Kürzung des Originalmanuskriptes.

\author{
Eingegangen im Februar 1979. \\ Reçu pour publication en février 1979.
}

\section{RÉSUMÉ}

On décrit la présence d'amylö̈de dans les organes internes de reines d'abeille vieillissantes. Ces composés glycoprotéiniques se trouvent sous la forme de petits corpuscules microscopiques, souvent agglomérés, en position intra- ou extracellulaire, dans les tissus les plus divers et on peut facilement les mettre en évidence par la méthode des indicateurs colorés dans des préparations globales ou sur des coupes.

Le matériel comprenait 97 reines âgées d'un à cinq ans. Les colorations spécifiques sont très simples :

\section{Réaction à l'iode}

Pré-traitement dans le Lugol, solution d'iode-iodure de potassium.

Post-traitement à l'acide sulfurique dilué.

2. Réaction PAS (acide périodique - réaction de Schiff)

3. Coloration au carmin

Pré-traitement à la diastase (élimination du glycogène).

Coloration au carmin de Best.

Post-coloration à l'hématoxyline de fer.

Jusqu'à présent on n'a jamais trouvé de dépôts amyloïdés chez de jeunes reines; ils sont par contre présents chez toutes les reines plus âgées, saines pour la plupart. Contrairement aux formations caverneuses déjà connues dans le système nerveux central (Fig. 1, Tabl. 1), qui ont été observées également chez des reines jeunes, il s'agit de modifications dues spécifiquement au vieillissement, comme c'est le cas pour les dépots de glycogène ou pour divers autres phénomènes cataboliques cellulaires.

A un âge élevé les corps amyloïdés peuvent atteindre un diamètre de $10 \mu \mathrm{m}$ et plus. Ils se situent aussi bien à l'intérieur des cellules qu'entre elles (Fig. 4, 8).

Les lieux de prédilection pour les dépôts amylö̈dés sont le système nerveux dans son ensemble, les cellules neurosécrétrices, les corpora cardiaca, les cellules adipeuses, les fibres musculaires, l'épithélium de la spermathèque. Chez des reines âgées entre autres, les corpora allata, l'intestin moyen, les ovaires, les glandes mandibulaires et la glande à venin étaient dépourvues d'amyloïde. 
On présume l'existence d'une relation entre l'amylö̈dose des cellules neurosécrétrices du ganglion cérébral et des corpora cardica et la production restreinte de substance royale chez les reines âgées d'une part, et entre les dépôts amyloïdés de l'épithélium de la spermathèque et le caractère bourdonneux des reines âgées d'autre part.

\section{LITERATURVERZEICHNIS}

BiedermanN M., 1964. - Neurosekretion bei Arbeitsbienen und Königinnen von Apis mellifica L. unter natürlichen und experimentellen Bedingungen. Z. wiss. Zool. 170, 256.

Borst M., 1950. - Pathologische Histologie. 4. Aufl. (Verlag J. F. Bergmann, München).

BUtLer C. G., 1960. - The significance of queen substance in swarming and supersedure in honeybee (Apis mellifera L.) colonies. Proc. R. Ent. Soc. London (4) 35, 129-132.

FYG W., 1956. - Merkmale zur Altersbestimmung von Bienenköniginnen. Schweiz. Bienenztg. 79, 428.

FIG W., 1960. - Über die Ablagerung von Amyloid im Samenblasenepithel der Bienenkönigin. $Z$. angew. Entomol. 45, 415.

FYG W., 1961. - Über die Glykogenspeicherung im Epithel der Valvula cardiaca der Honigbiene (Apis mellifica L.) Z. Bienenforsch. 5, 213.

FYG W., 1963. - Anomalien und Krankheiten der Bienenkönigin. Bull. apicole 6, 7.

FyG W., 1964. - Anomalies and diseases of the queen honey bee. Ann. Rev. Entomol. 9, 207.

FYG W., 1965. - Über die Lokalisation des Glykogens in den larvalen und pupalen Fettkörperzellen der Honigbiene (Apis mellifica L.) Z. Bienenforsch. 8, 55.

FyG W., 1966. - Über den Bau und die Funktion der Valvula vaginalis der Bienenkönigin (Apis mellifica L.) Z. Bienenforsch. 8, 256.

FYG W., 1967. - Über verschiedene Zelleinschlüsse im Pylorusepithel der Bienenkönigin (Apis mellifica L.) Z. Bienenforsch. 9, 171.

Herrmann H., 1969. - Die neurohormonale Kontrolle der Paarungsflüge und der Eilegetätigkeit bei der Bienenkönigin. Z. Bienenforsch. 9, 509.

Koeniger G., 1970. - Bedeutung der Tracheenhülle und der Anhangsdrüse der Spermatheka für die Befruchtungsfähigkeit der Spermatozoen in der Bienenkönigin (Apis mellifica L.) Apidologie 1, 5571.

LuCht-Bertram E., 1963. - Degenerative Erscheinungen am Gehirn alternder Bienenköniginnen. $Z$. Bienenforsch. 6, 169.

Örösı-PAL Z., 1968. - Physiologie des glandes nourricières, in Chauvin R. : Traité de Biologie de l'A beille 1, 272.

Pflugfelder O., 1958. - Entwicklungsphysiologie der Insekten. 2. Aufl. (Akad. Verlagsges. Geest und Portig, Leipzig).

RoMeIS B., 1948. - Mikroskopische Technik R. Oldenbourg München 1948.

SCHmidT H., 1923. - Über den Alterstod der Biene. Z. Naturwiss. (Jena) 59.

Schneider G., 1964. - Über die Pathogenese der Amyloidose. Immunologische, histochemische und morphologische Untersuchungen. Ergebn. Path. 44, 1.

Schwartz Ph., 1966. - New aspects of presenile and senile Deterioration. A new triad : cerebral, cardiovascular and pancreatic insular amyloidosis of aged. 7th Intern. Congr. Gerontology, Vienna.

WEYER F., 1931. - Cytologische Untersuchungen am Gehirn alternder Bienen und die Frage nach dem Alterstod. Z. Zellforsch. und mikr. Anat. 14, 1. 